TRANSACTIONS OF THE

AMERICAN MATHEMATICAL SOCIETY

Volume 351, Number 6, Pages 2539-2551

S 0002-9947(99)02462-9

Article electronically published on February 15, 1999

\title{
REPRESENTATION THEORY OF REDUCTIVE NORMAL ALGEBRAIC MONOIDS
}

\author{
STEPHEN DOTY
}

\begin{abstract}
New results in the representation theory of "semisimple" algebraic monoids are obtained, based on Renner's monoid version of Chevalley's big cell. (The semisimple algebraic monoids have been classified by Renner.) The rational representations of such a monoid are the same thing as "polynomial" representations of the associated reductive group of units in the monoid, and this representation category splits into a direct sum of subcategories by "homogeneous" degree. We show that each of these homogeneous subcategories is a highest weight category, in the sense of Cline, Parshall, and Scott, and so equivalent with the module category of a certain finite-dimensional quasihereditary algebra, which we show is a generalized Schur algebra in S. Donkin's sense.
\end{abstract}

\section{Introduction}

Let $M$ be an affine algebraic monoid over an algebraically closed field $K$. Assuming $M$ is reductive, i.e. its group $G$ of units is a reductive group, what can one say about the representation theory of $M$ over $K$ ? This is the main question considered here. But as it stands it is still too general; here we confine our attention to just those $M$ which are (irreducible) normal varieties.

There are two good reasons for assuming our monoid $M$ is normal. First, recall that any affine algebraic group is smooth and hence normal. The normality of the algebraic group plays a significant role in its representation theory, for instance in the proof of Chevalley's theorem classifying the irreducible representations. Thus it seems reasonable in trying to extend representation theory from reductive groups to reductive monoids to look first at the case when $M$ is normal. Second, L. Renner [14] has obtained a classification theorem for such monoids under the additional assumptions that the center $Z(M)$ is 1-dimensional and that $M$ has a zero element. Renner calls such algebraic monoids semisimple and proves that they are classified by data of the form $(X(T), \Phi, X(\bar{T}))$, where $(X(T), \Phi)$ is the usual root data which classifies the reductive group $G$ and $X(\bar{T})$ is the character group of the closure (in $M)$ of the maximal torus $T$.

Renner's classification theorem depends on an algebraic monoid version of Chevalley's big cell, which holds for any reductive affine algebraic monoid (with no assumptions about its center or a zero). As a corollary of its construction, Renner derives a very useful "extension principle" [14, (4.5) Corollary] which is the main technical tool for the present work.

Received by the editors June 26, 1996 .

1991 Mathematics Subject Classification. Primary 20G05, 20M30; Secondary 16G99, 22E55.

Partially supported by NSF grant DMS-9401576. 
It is worth noting that it is very easy to construct reductive algebraic monoids. Just take a matrix representation $\rho: G \rightarrow \mathrm{GL}_{n}(K)$ of some reductive affine algebraic group $G$ and set $M=M(\rho)=\overline{\rho(G)}$, the closure in $\mathrm{M}_{n}(K)$. Then $M$ will be a reductive algebraic monoid. If instead we begin with a semisimple $G$ and then set $M$ equal to the closure of $K^{\times} \rho(G)$ then $\operatorname{dim} Z(M)$ will be 1 and $M$ will still be reductive. In either case we can then pass to the normalization of $M$; the resulting monoid will be a reductive normal algebraic monoid.

Our interest in representations of algebraic monoids is an outgrowth of our interest in polynomial representations of algebraic groups. As pointed out in [8], whenever we represent an affine algebraic group $G$ by matrices then we can formulate the notion of a polynomial representation (one that depends polynomially on the matrix coordinates) of $G$ and then the polynomial representation theory of $G$ is precisely the same as the rational representation theory of the associated algebraic monoid $M=\bar{G}$. If (the representation of) $G$ contains the scalar matrices, then the polynomial representations split into homogeneous components and the representation theory in a given homogeneous degree $d$ is completely equivalent with that of a certain associated finite-dimensional algebra $S_{d}(G)$ [8]. Of course, this is dependent on the choice of matrix representation.

Let us enumerate the main results of the paper. First, we show that the restriction functor from an algebraic monoid to a closed submonoid admits a right adjoint, which we call induction. All the usual elementary properties of the induction functor hold in this setting, excepting the tensor identity. We then prove the following generalization of a recent result of Friedlander-Suslin [9]: if $V$ is a rational $G$-module all the weights of which are polynomial, then $V$ lifts uniquely to a rational $M$-module. From this we obtain a classification of the simple rational $M$-modules by highest weight. We then study truncation functors following S. Donkin [4] and show that the coordinate algebra $K[M]$ has a good filtration. From this it follows that the category of rational $M$-modules is a highest weight category in the sense of Cline-Parshall-Scott [2]. We then consider the case where $M$ has a (faithful) matrix representation with the property that its restriction to its group of units $G$ admits a graded polynomial representation theory in the sense of [8]. Our final result is that in such a case the "Schur" algebras $S_{d}(G)$ defined in [8] are generalized Schur algebras in Donkin's sense [4] and so they must be quasihereditary. In particular, they have finite global dimension.

I would like to thank S. Donkin for pointing out an error in an earlier version of this paper.

\section{INDUCED Modules}

If $G$ is an affine algebraic group and $H$ a closed subgroup of $G$ then, we have the restriction functor $\operatorname{res}_{H}^{G}$ from rational $G$-modules to rational $H$-modules. This functor admits a right adjoint ind ${ }_{H}^{G}$, induction from $H$ to $G$. Since adjoint functors, if they exist, are unique up to natural isomorphism, this property determines the induction functor.

Now suppose $M$ is an affine algebraic monoid and $N$ a closed submonoid. Then the restriction functor $\operatorname{res}_{N}^{M}$ still admits a right adjoint and we continue to denote it by $\operatorname{ind}_{N}^{M}$. While this is basically trivial, still one needs to check which properties will carry over from groups to monoids. It turns out that Frobenius reciprocity carries over but the tensor identity does not. 
Given two varieties $X, Y$ we denote by $\operatorname{Mor}(X, Y)$ the space of all morphisms $X \rightarrow Y$. Let $V$ be a vector space over $K$. Given any $m$ in $M$ and any $f$ in $\operatorname{Mor}(M, V)$, there exist maps $L_{m} f$ and $R_{m} f$ in $\operatorname{Mor}(M, V)$ (resp., left and right translation) defined by the rules

$$
L_{m} f(x)=f(m x), \quad R_{m} f(x)=f(x m), \quad \text { all } x \text { in } M .
$$

The action $(f, m) \mapsto L_{m} f$ is a right action and $(m, f) \mapsto R_{m} f$ a left one. Obviously these actions of $M$ on $\operatorname{Mor}(M, V)$ commute: $R_{m}\left(L_{m^{\prime}} f\right)=L_{m^{\prime}}\left(R_{m} f\right)$ for $m, m^{\prime}$ in $M$. Taking $V=K$ in the above we obtain corresponding actions of $M$ on $K[M]=\operatorname{Mor}(M, K)$.

Suppose now that $V$ is a rational $N$-module. We define the corresponding induced module $\operatorname{ind}_{N}^{M} V$ by

$$
\{f \in \operatorname{Mor}(M, V): f(n x)=n f(x) \text {, all } x \in M, n \in N\} .
$$

This becomes a rational $M$-module via the action $(m, f) \mapsto R_{m} f$, since $\left(R_{m} f\right)(n x)$ $=f(n x m)=n f(x m)=n\left(R_{m} f\right)(x)$.

Given any homomorphism $\varphi: V \rightarrow V^{\prime}$ between two rational $N$-modules $V$, $V^{\prime}$ we have a corresponding induced homomorphism $\operatorname{ind}_{N}^{M} \varphi: \operatorname{ind}_{N}^{M} V \rightarrow \operatorname{ind}_{N}^{M} V^{\prime}$ given by composition with $\varphi$ : $\left.\operatorname{(ind}_{N}^{M} \varphi\right)(f)=\varphi \circ f$.

Let $\varepsilon_{V}: \operatorname{Mor}(M, V) \rightarrow V$ denote the evaluation map, given by $f \mapsto f(1)$. By restriction this gives a map, also denoted by $\varepsilon_{V}$, from $\operatorname{ind}_{N}^{M} V$ to $V$. It is trivial to check that evaluation $\varepsilon_{V}: \operatorname{ind}_{N}^{M} V \rightarrow V$ is a homomorphism of rational $N$-modules.

We now list some basic properties of induction.

2.1. Universal mapping. Given any rational $M$-module $U$ and a homomorphism of rational $N$-modules $\varphi: U \rightarrow V$ there exists a unique homomorphism $\tilde{\varphi}: U \rightarrow$ $\operatorname{ind}_{N}^{M} V$ of rational $M$-modules such that $\varphi=\varepsilon_{V} \circ \tilde{\varphi}$.

Indeed, given $u \in U$, let $\tilde{\varphi}(u)$ be the element of $\operatorname{Mor}(M, V)$ such that $\tilde{\varphi}(u)(x)=$ $\varphi(x u)$ for all $x \in M$. It is easy to check that $\tilde{\varphi}(u)$ lies $\operatorname{in} \operatorname{ind}_{N}^{M} V$ and that $\varphi=\varepsilon_{V} \circ \tilde{\varphi}$. The uniqueness statement is left to the reader.

2.2. Frobenius reciprocity. $\operatorname{Hom}_{M}\left(U, \operatorname{ind}_{N}^{M} V\right) \simeq \operatorname{Hom}_{N}\left(\operatorname{res}_{N}^{M} U, V\right)$.

In fact, the correspondence is given by $\tilde{\varphi} \leftrightarrow \varphi$. The next fact is now obvious.

2.3. Adjointness. The functor $\operatorname{ind}_{N}^{M}$ is right adjoint to the functor $\operatorname{res}_{N}^{M}$.

2.4. Exactness. The functor $\operatorname{ind}_{N}^{M}$ is left exact.

This follows from the adjointness property, as does the next property.

2.5. Transistivity. If $N<L<M$ is a chain of closed monoid inclusions we have an isomorphism of functors between $\operatorname{ind}_{N}^{M}$ and $\operatorname{ind}_{L}^{M} \circ \operatorname{ind}_{N}^{L}$.

However, the tensor identity fails to hold for induction in this generality; see [7, $5.10(\mathrm{~b})]$ for a counterexample.

Suppose now that $M, N$ both have an algebraic group structure. Then the induced module $\operatorname{ind}_{N}^{M} V$ as defined in (2) is isomorphic with

$$
\left\{f \in \operatorname{Mor}(M, V): f(x n)=n^{-1} f(x), \text { all } x \in M, n \in N\right\}
$$

which is made into a rational $M$-module by the action $(m, f) \mapsto L_{m^{-1}} f$. 
Recall that $V \otimes K[M]$ is isomorphic with $\operatorname{Mor}(M, V)$; the isomorphism takes $v \otimes f$ to the morphism $x \mapsto f(x) v$. Now $V \otimes K[M]$ can be made into a left $N$-module via $(n, v \otimes f) \mapsto(n v) \otimes\left(R_{n} f\right)$. Then the set of $N$-invariants

$$
(V \otimes K[M])^{N}
$$

becomes a right $M$-module with $M$ acting by $(v \otimes f, m) \mapsto v \otimes L_{m} f$. If $M$ is only a monoid that is in general the best we can do, but if $M$ has a group structure we can make (4) a left $M$-module by composing with the anti-involution $m \mapsto m^{-1}$. Specifically, the left action is given by $(m, v \otimes f) \mapsto v \otimes L_{m^{-1}} f$. If $N$ also has a group structure then the $M$-modules in (2), (3), and (4) are all isomorphic.

Thus we see that the tensor description (4) of the induced module, the usual definition in the group situation, is not appropriate to the monoid case. This is responsible for the failure of the tensor identity to generalize.

\section{Representation Theory}

To reiterate, $K$ is always an algebraically closed field. It is well-known (see [3]) that any affine algebraic monoid $M$ over $K$ is isomorphic with a closed submonoid of the monoid $\mathrm{M}_{n}=\mathrm{M}_{n}(K)$ of $n \times n$ matrices over $K$; i.e., $M$ has a faithful matrix representation. One can refer to Putcha [13] and Solomon [16] for basic properties of algebraic monoids. We will always assume $M$ is irreducible as a variety. Let $G$ be the group of units of $M$. Then $G$ is a connected linear algebraic group defined over $K$ and $G$ is dense in $M$.

Assume from now on that $M$ is reductive. Choose a maximal torus $T$ in $G$ and let $\bar{T}$ denote the Zariski closure of $T$ in $M$. Let

$$
X(T)=\operatorname{Hom}\left(T, K^{\times}\right)
$$

denote the group of characters (i.e., algebraic group morphisms from $T$ to the multiplicative group $K^{\times}$) on $T$ and

$$
X(\bar{T})=\operatorname{Hom}(\bar{T}, K)
$$

the monoid of characters (i.e., algebraic monoid morphisms from $\bar{T}$ to the multiplicative monoid $K)$ on $\bar{T}$. The restriction $\left.\lambda\right|_{T}$ of a character $\lambda \in X(\bar{T})$ is a monoid character mapping $T$ to $K$. Since $T$ is a group it is automatic that $\left.\lambda\right|_{T}$ is a group character and that its image is contained in $K^{\times}$, so $\lambda_{T} \in X(T)$. Note that only the identity character on $\bar{T}$ restricts to the identity character on $T$. Thus, the restriction map $X(\bar{T}) \rightarrow X(T)$ is injective. In all that follows, it will be convenient to identify $X(\bar{T})$ with its image in $X(T)$. We will refer to elements of $X(T)$ as rational weights and to elements of $X(\bar{T})$ as polynomial weights.

Now suppose $V$ is a rational $\bar{T}$-module. For $\lambda \in X(\bar{T})$ we have the weight space

$$
V_{\lambda}=\{v \in V: t v=\lambda(t) v, \quad \text { all } t \in \bar{T}\} .
$$

By the usual argument $V$ is the direct sum of its weight spaces: $V=\bigoplus_{\lambda \in X(\bar{T})} V_{\lambda}$. Note that the $T$-weight spaces of $V$ are the same as the $\bar{T}$-weight spaces of $V$. When we speak of the weight spaces of a rational $M$-module $V$ we mean its weight spaces relative to its restriction to $\bar{T}$.

If $V$ is a rational $M$-module then the weights of its restriction to $G$ are all polynomial weights. If we assume $M$ is normal then the converse holds. 
3.1. Theorem. Suppose $M$ is a normal reductive affine algebraic monoid. Let $V$ be a rational $G$-module all the $T$-weights of which are polynomial. Then the $G$-module structure on $V$ extends uniquely to a rational $M$-module structure.

Proof. Choose a basis $\left(v_{i}\right)_{i \in I}$ of $T$-weight vectors for $V$. Since the weights are all polynomial there exist elements $\lambda_{j}$ in $X(\bar{T})$ for each $j \in I$ such that

$$
t v_{j}=\lambda_{j}(t) v_{j}, \quad \text { all } t \in \bar{T} .
$$

For $j \in I$ let $c_{i j} \in K[G]$ be defined by

$$
g v_{j}=\sum_{i \in I} c_{i j}(g) v_{i}, \quad g \in G .
$$

The $K$-span of the $c_{i j}$ is the coefficient space $\operatorname{cf}_{G}(V)$.

For each $i, j \in I$ let us define $\tilde{c}_{i j}: \bar{T} \rightarrow K$ by

$$
\tilde{c}_{i j}= \begin{cases}\lambda_{j} & \text { if } i=j, \\ 0 & \text { otherwise. }\end{cases}
$$

Clearly $\tilde{c}_{i j}$ and $c_{i j}$ have the same restriction to $T$. Thus, by Renner's extension principle $[14,(4.5)]$, there exist unique $c_{i j}^{\prime} \in K[M]$ such that $c_{i j}^{\prime}$ coincides with $c_{i j}$ on restriction to $G$ and coincides with $\tilde{c}_{i j}$ on restriction to $\bar{T}$. Then the equations

$$
m v_{j}=\sum_{i \in I} c_{i j}^{\prime}(m) v_{i}, \quad m \in M,
$$

define the desired rational $M$-module structure on $V$.

3.2. Remark. The conclusion of the previous theorem in the special case $M=\mathrm{M}_{n}$ is the content of Friedlander-Suslin [9, Prop. 3.4].

Now choose a Borel subgroup $B$ of $G$ with $T \subset B$. Let $\Phi=\Phi(G, T)$ be the set of roots associated to the pair $(G, T)$ and set $\Phi^{-}=\Phi(B, T), \Phi^{+}=-\left(\Phi^{-}\right)$. Let $\Delta$ denote the set of simple roots relative to $\Phi^{+}$and set $W=N_{G}(T) / T$, the Weyl group associated to the pair $(G, T)$.

The set of dominant rational weights is the set $X(T)^{+}$, consisting of those $\lambda \epsilon$ $X(T)$ for which $\left\langle\lambda, \alpha^{\vee}\right\rangle \geq 0$ for all $\alpha \in \Phi^{+}$. The set of dominant polynomial weights is the set

$$
X(\bar{T})^{+}=X(\bar{T}) \cap X(T)^{+} .
$$

Recall that the set $X(T)$ is partially ordered by declaring $\lambda \leq \mu$ if and only if $\lambda-\mu$ can be written as a nonnegative integral linear combination of positive roots. This of course induces a partial order on the set $X(T)^{+}$.

For $\lambda \in X(T)^{+}$, let $L(\lambda)$ denote the simple rational $G$-module of highest weight $\lambda$. Every simple rational $G$-module is isomorphic to $L(\lambda)$ for some dominant rational weight $\lambda$; moreover, $\lambda \neq \mu$ implies $L(\lambda) \not ॅ L(\mu)$.

For $\lambda \in X(T)$ denote by $K_{\lambda}$ the one-dimensional $T$-module affording the character $\lambda$. Recall that $B=T U$ where $U$ is its unipotent radical. In particular, every $b \in B$ is uniquely expressible in the form $b=t u$ with $t \in T, u \in U$, and we have a morphism of algebraic groups $B \rightarrow T$ defined by $b \mapsto t$. The action of $T$ on $K_{\lambda}$ extends to a rational $B$-action by composition with the above morphism $B \rightarrow T$. Let $H^{0}(\lambda)=\operatorname{ind}_{B}^{G} K_{\lambda}$ be the corresponding induced $G$-module. Then $H^{0}(\lambda)$ is nonzero if and only if $\lambda$ is dominant and in that case we have $L(\lambda)=\operatorname{soc}_{G} H^{0}(\lambda)$. 
Now suppose $\lambda \in X(\bar{T})$ and let $K_{\lambda}$ denote the one-dimensional $\bar{T}$-module affording the character $\lambda$. We need the following.

3.3. Lemma. The morphism $B \rightarrow T$ described above extends to an algebraic monoid morphism $\bar{B} \rightarrow \bar{T}$.

Proof. We may identify $M$ with a closed submonoid of $\mathrm{M}_{n}$, in such a way that $T$ consists of diagonal matrices and $B$ consists of upper triangular matrices [11, Prop. 15.4 and Thm. 17.6]. Then the same is true of $\bar{T}$ and $\bar{B}$. In this guise the map $B \rightarrow T$ is given by the rule

$$
\left[\begin{array}{ccc}
a_{11} & \ldots & a_{1 n} \\
& \ddots & \vdots \\
& & a_{n n}
\end{array}\right] \mapsto\left[\begin{array}{ccc}
a_{11} & & \\
& \ddots & \\
& & a_{n n}
\end{array}\right] .
$$

Now consider the algebraic group $\mathrm{D}_{n}$ of all diagonal matrices in $\mathrm{GL}_{n}$ and the algebraic group $\mathrm{T}_{n}$ of all upper triangular matrices in $\mathrm{GL}_{n}$. The closure $\overline{\mathrm{D}}_{n}$ in $\mathrm{M}_{n}$ consists of all diagonal matrices in $\mathrm{M}_{n}$ and $\overline{\mathrm{T}}_{n}$ consists of all upper triangular matrices in $\mathrm{M}_{n}$. The map $\varphi: \overline{\mathrm{T}}_{n} \rightarrow \overline{\mathrm{D}}_{n}$ defined by the same rule (6) is a morphism of algebraic monoids and its restriction to $\bar{B}$ is the desired extension.

By composing with the morphism $\bar{B} \rightarrow \bar{T}$ we can extend $K_{\lambda}$ to a rational $\bar{B}$ module. The induced module $\operatorname{ind} \frac{M}{B} K_{\lambda}$ is a rational $M$-module. Since the restriction map $K[M] \rightarrow K[G]$ is injective (see $[8,2.1]$ ) we observe that ind $\frac{M}{B} K_{\lambda}$ is isomorphic with a submodule of $H^{0}(\lambda)=\operatorname{ind}_{B}^{G} K_{\lambda}$. Hence, a necessary condition for $\operatorname{ind} \frac{M}{B} K_{\lambda}$ to be nonzero is that $\lambda \in X(\bar{T})^{+}$. Thanks to Renner's extension principle, we can establish the converse as well.

3.4. Theorem. Let $M$ be a normal reductive affine algebraic monoid. Let $\lambda \in$ $X(\bar{T})$. The induced module ind $\frac{M}{B} K_{\lambda}$ is nonzero if and only if $\lambda$ lies in $X(\bar{T})^{+}$.

Proof. If $\operatorname{ind} \frac{M}{B} K_{\lambda} \neq(0)$ then it has a $G$-submodule isomorphic with $L(\lambda)$, because ind $\frac{M}{B} K_{\lambda}$ embeds as a $G$-submodule of $H^{0}(\lambda)$. Thus the $\lambda$-weight space of $\operatorname{ind} \frac{M}{B} K_{\lambda}$ is nontrivial. But all the weights of a rational $M$-module are polynomial, consequently $\lambda$ is polynomial. Moreover, $\lambda$ is dominant since $H^{0}(\lambda) \neq(0)$. Thus $\lambda \in X(\bar{T})^{+}$.

Conversely, suppose $\lambda \in X(\bar{T})^{+}$. Then $H^{0}(\lambda)=\operatorname{ind}_{B}^{G} K_{\lambda} \neq(0)$. Thus there exists some $f \in K[G]$ satisfying

$$
f(b g)=\lambda(b) f(g), \quad \text { all } b \in B, g \in G .
$$

Thus we have the equality $\left.f\right|_{T}=\left.a \lambda\right|_{T}$, where $a=f(1)$ and where we regard $\lambda$ as a character on $\bar{T}$. By the extension principle [14, (4.5)], there exists a unique $\tilde{f} \in K[M]$ such that $\left.\tilde{f}\right|_{G}=f$ and $\left.\tilde{f}\right|_{T}=a \lambda$.

To complete the proof we need only show that $\tilde{f}$ satisfies the condition

$$
\tilde{f}(b m)=\lambda(b) \tilde{f}(m), \quad \text { all } b \in \bar{B}, m \in M .
$$

Since $\left.\tilde{f}\right|_{G}=f$ we have the equality (8) at least for $b \in B$ and $m \in G$. Holding $b \in B$ fixed, observe that the map

$$
m \mapsto \tilde{f}(b m)-\lambda(b) \tilde{f}(m)
$$


in $K[M]$ is zero on the open set $G$. Thus it is zero on $M$; i.e., we have the equality (8) for all $b \in B, m \in M$. Now hold $m \in M$ fixed and consider the map

$$
b \mapsto \tilde{f}(b m)-\lambda(b) \tilde{f}(m)
$$

in $K[\bar{B}]$. Since this map vanishes on the open set $B$, it vanishes on $\bar{B}$. This establishes (8) for all $b \in \bar{B}, m \in M$.

Now we obtain the highest-weight classification of the simple $M$-modules. This result was already obtained in the case when $\operatorname{dim} Z(M)=1$ and $M$ has a zero element in Renner [15, Theorem 5.2].

3.5. Corollary. Let $M$ be a normal reductive affine algebraic monoid. The set of isomorphism classes of simple rational $M$-modules is $\left\{L(\lambda): \lambda \in X(\bar{T})^{+}\right\}$.

Proof. Let $V$ be a simple rational $M$-module. Then $V$ remains simple on restriction to $G$, so $V \simeq L(\lambda)$ for some $\lambda \in X(T)^{+}$. Clearly $\lambda$ is polynomial, so in fact $\lambda \in X(\bar{T})^{+}$.

On the other hand, suppose $\lambda \in X(\bar{T})^{+}$. Then $\operatorname{ind} \frac{M}{B} K_{\lambda} \neq(0)$ and as already argued it follows that $L(\lambda)$ is a $G$-submodule of $\operatorname{ind} \frac{M}{B} K_{\lambda}$. Therefore all its weights are polynomial. By $3.1, L(\lambda)$ lifts to a rational $M$-module.

We can now clarify the relation between $X(\bar{T})^{+}$and $X(T)^{+}$.

3.6. Proposition. Let $T$ be a maximal torus in the group $G$ of units in a reductive normal algebraic monoid $M, \bar{T}$ its closure in $M$. Assume that the center $Z(M)$ is 1-dimensional and that $M$ has a zero element. Then the set $X(\bar{T})^{+}$is an ideal (i.e. a "saturated" subset in the language of Donkin [4]) in the poset $X(T)^{+}$.

Proof. According to Renner's classification theorem [14, (6.5) Theorem], $M$ is determined up to isomorphism over $K$ by the datum $(X(T), \Phi, X(\bar{T}))$. Let $M_{\mathbb{C}}$ be the corresponding reductive monoid over the complex field $\mathbb{C}$ and let $G_{\mathbb{C}}, B_{\mathbb{C}}, T_{\mathbb{C}}$, etc. be the groups over $\mathbb{C}$ corresponding with $G, B, T$. Then of course $X\left(T_{\mathbb{C}}\right)=X(T)$, $X(\bar{T})=X\left(\overline{T_{\mathbb{C}}}\right), X\left(T_{\mathbb{C}}\right)^{+}=X(T)^{+}, X(\bar{T})^{+}=X\left(\overline{T_{\mathbb{C}}}\right)^{+}$. Thus it suffices to prove the stated result in the case $K=\mathbb{C}$.

Assuming now that $K=\mathbb{C}$, let $\lambda \leq \mu$ for $\lambda \in X(T)^{+}$and $\mu \in X(\bar{T})^{+}$. We must show that $\lambda \in X(\bar{T})^{+}$. By the reductive version of [10,21.3], $\lambda$ is a weight of the irreducible module of highest weight $\mu$ for the Lie algebra $\mathfrak{g}$ of $G$, thus $\lambda$ is a weight of $L(\mu)$. Hence $\lambda$ is polynomial.

\section{Truncation Functors}

A good filtration for a rational $G$-module $V$ is an ascending series

$$
(0)=V_{0} \subset V_{1} \subset V_{2} \subset \cdots
$$

of $G$-submodules of $V$ such that $V$ is the union of the $V_{i}$ and such that for each $i$ there exists some $\lambda_{i} \in X(T)^{+}$for which $V_{i+1} / V_{i} \simeq H^{0}\left(\lambda_{i}\right)$. The number of subquotients $V_{i+1} / V_{i}$ isomorphic to a given $H^{0}(\lambda)$ is denoted by $\left(V: H^{0}(\lambda)\right)$. Note that this may be infinite.

We keep all the notations and assumptions of the previous section. In particular, $M$ is a normal reductive monoid with unit group $G, T$ a maximal torus in $G$, and $B$ a Borel subgroup containing $T$. We will suppose additionally that $M$ has 1dimensional center $Z(M)$ and that $M$ has a zero element, so we are from now on dealing with one of Renner's so-called "semisimple" monoids. We are going to show 
that $\operatorname{ind} \frac{M}{B} K_{\lambda}$ is isomorphic with $H^{0}(\lambda)=\operatorname{ind}_{B}^{G} K_{\lambda}$ for all $\lambda \in X(\bar{T})^{+}$. From this it will follow that the principal injective objects in the category of rational $M$-modules all have good filtrations and those filtrations satisfy the expected reciprocity law. From this in turn we see that $K[M]$ has a good filtration and $\left(K[M]: H^{0}(\lambda)\right)=$ $\operatorname{dim} H^{0}(\lambda)$ for all $\lambda \in X(\bar{T})^{+}$.

From Donkin [4] we have two functors on rational $G$-modules. To describe (a special case of) the first functor, let $V$ be a rational $G$-module. Since $G$ is dense in $M$, the restriction map $K[M] \rightarrow K[G]$ is injective. Thus we may identify $K[M]$ with a subcoalgebra of $K[G]$. There is a unique maximal $G$-submodule of $V$ having the property that its coefficient space lies in $K[M]$ : the union of all submodules with that property. Denoting this submodule by $F_{M} V$ we get a functor $V \mapsto F_{M} V$ from rational $G$-modules to rational $M$-modules. We may view $F_{M} V$ as the maximal $G$-submodule of $V$ which extends to a rational $M$-module.

The second functor depends on choosing a subset $\pi \subset X(T)^{+}$. If $V$ is a rational $G$-module, one says that $V$ belongs to $\pi$ if all the composition factors of $V$ have highest weights lying in $\pi$. Set $O_{\pi} V$ equal to the unique maximal $G$-submodule of $V$ belonging to $\pi$. The assignment $V \mapsto O_{\pi} V$ is the second functor. One may ask how these functors relate when $\pi$ is taken to be the set $X(\bar{T})^{+}$of dominant polynomial weights. The answer is provided by the following.

4.1. Lemma. With $\pi=X(\bar{T})^{+}$we have the equality $O_{\pi}=F_{M}$.

Proof. Let $V$ be a rational $G$-module. The inclusion $F_{M} V \subset O_{\pi} V$ is clear, since by 3.5 the composition factors of $F_{M} V$ all belong to $\pi$. On the other hand, by 3.1 we know that $O_{\pi} V$ lifts to a rational $M$-module, establishing the opposite inclusion $O_{\pi} V \subset F_{M} V$.

4.2. Theorem. Suppose the reductive normal algebraic monoid $M$ has 1-dimensional center $Z(M)$ and that $M$ has a zero element. Let $\pi=X(\bar{T})^{+}$. For any $\lambda \in X(T)^{+}$we have

$$
O_{\pi} H^{0}(\lambda)= \begin{cases}H^{0}(\lambda) & \text { if } \lambda \in \pi, \\ (0) & \text { otherwise. }\end{cases}
$$

Moreover, for $\lambda \in \pi$ we have an isomorphism between $H^{0}(\lambda)$ and $\operatorname{ind} \frac{M}{B} K_{\lambda}$.

Proof. Since $H^{0}(\lambda)$ has simple socle $L(\lambda)$, any nonzero submodule of $H^{0}(\lambda)$ must also have socle $L(\lambda)$. Thus $O_{\pi} H^{0}(\lambda)=(0)$ if $\lambda \notin \pi$.

Now suppose that $\lambda \in \pi$. Since the highest weights of the composition factors of $H^{0}(\lambda)$ are all $\leq \lambda$, by 3.6 we have the equality $O_{\pi} H^{0}(\lambda)=H^{0}(\lambda)$. Moreover, since ind $\frac{M}{B} K_{\lambda}$ embeds in $H^{0}(\lambda)$, there is an $M$-submodule of $H^{0}(\lambda)$ isomorphic to $\operatorname{ind} \frac{M}{B} K_{\lambda}$. This also shows that there is a unique $\bar{B}$-stable line in $F_{M} H^{0}(\lambda)$. Therefore, by Frobenius reciprocity, there is a nontrivial homomorphism $F_{M} H^{0}(\lambda) \rightarrow \operatorname{ind} \frac{M}{B} K_{\lambda}$. Since both sides have the same socle, this is injective. Hence $\operatorname{dim} F_{M} H^{0}(\lambda) \leq \operatorname{dimind} \frac{M}{B} K_{\lambda}$. It follows that $\operatorname{ind} \frac{M}{B} K_{\lambda} \simeq F_{M} H^{0}(\lambda)$. Since $F_{M} H^{0}(\lambda)=O_{\pi} H^{0}(\lambda)=H^{0}(\lambda)$, the proof is complete.

For $\lambda \in X(T)^{+}$, let $I(\lambda)$ be the injective envelope of $L(\lambda)$ in the category of rational $G$-modules. Similarly, for $\lambda \in X(\bar{T})^{+}$, let $Q(\lambda)$ be the injective envelope of $L(\lambda)$ in the category of rational $M$-modules. Denote by $[V: L]$ the multiplicity of a simple module $L$ in a composition series of $V$. 
4.3. Theorem. Suppose the reductive normal algebraic monoid $M$ has 1-dimensional center $Z(M)$ and that $M$ has a zero element. Let $\pi=X(\bar{T})^{+}$. For any $\lambda \in X(T)^{+}$we have

$$
O_{\pi} I(\lambda)= \begin{cases}Q(\lambda) & \text { if } \lambda \in \pi \\ (0) & \text { otherwise }\end{cases}
$$

Moreover, for $\lambda \in \pi, Q(\lambda)$ has a good filtration satisfying the reciprocity law

$$
\left(Q(\lambda): H^{0}(\mu)\right)=\left[H^{0}(\mu): L(\lambda)\right] \quad \text { for all } \mu \in \pi
$$

Proof. Since $F_{M}$ takes injectives to injectives [4, (1.1d)], $F_{M} I(\lambda)=O_{\pi} I(\lambda)$ is injective as rational $M$-module. It is nonzero precisely when $\lambda \in \pi$ and in that case has simple socle $L(\lambda)$. This proves the first statement. The reciprocity statement follows from [6, Thm. 8].

4.4. Theorem. Suppose the reductive normal algebraic monoid $M$ has 1-dimensional center $Z(M)$ and that $M$ has a zero element. With $\pi=X(\bar{T})^{+}$, we have the equality $O_{\pi} K[G]=K[M]$. Moreover, $K[M]$ has a good filtration satisfying

$$
\left(K[M]: H^{0}(\mu)\right)=\operatorname{dim} H^{0}(\mu) \quad \text { for all } \mu \in \pi .
$$

Proof. Considering $K[M]$ as right $M$-module via left translation, we have $K[M]=$ $\bigoplus_{\lambda \in X(\bar{T})} K[M]_{\lambda}$. Similarly, $K[G]=\bigoplus_{\lambda \in X(T)} K[G]_{\lambda}$. These are weight spaces for the right action by left translation; since left and right translation commute they are also left modules for $M, G$, resp. Moreover, from the definition of induction we have isomorphisms $K[M]_{\lambda} \simeq \operatorname{ind} \frac{M}{T} K_{\lambda}$ and $K[G]_{\lambda} \simeq \operatorname{ind}_{T}^{G} K_{\lambda}$. Both $\operatorname{ind} \frac{M}{T} K_{\lambda}$ and $\operatorname{ind}_{T}^{G} K_{\lambda}$ are injective in their respective category, and by Frobenius reciprocity we have

$$
\left(\operatorname{ind}_{\bar{T}}^{M} K_{\lambda}: Q(\mu)\right)=\operatorname{dim} L(\mu)_{\lambda}, \quad \lambda \in X(\bar{T}), \mu \in X(\bar{T})^{+}
$$

and

$$
\left(\operatorname{ind}_{T}^{G} K_{\lambda}: I(\mu)\right)=\operatorname{dim} L(\mu)_{\lambda}, \quad \lambda \in X(T), \mu \in X(T)^{+},
$$

the symbol on the left-hand side of each equation denoting the number of summands isomorphic to the respective principal injective module. Thus for all $\lambda \in X(\bar{T}), \mu \in$ $X(\bar{T})^{+}$we have the equality

$$
\left(\operatorname{ind}_{\bar{T}}^{M} K_{\lambda}: Q(\mu)\right)=\left(\operatorname{ind}_{T}^{G} K_{\lambda}: I(\mu)\right) .
$$

It follows that $O_{\pi}$ takes $K[G]_{\lambda}$ onto $K[M]_{\lambda}$ for all $\lambda \in X(\bar{T})$ and to $(0)$ otherwise. Thus $O_{\pi} K[G] \simeq K[M]$.

Since $K[G]$ has a good filtration satisfying

$$
\left(K[G]: H^{0}(\lambda)\right)=\operatorname{dim} H^{0}(\lambda), \quad \lambda \in X(T)^{+}
$$

(see [6, Thm. 5]), the second statement of the theorem follows from [6, Thm. 8].

We also have the following corollary to the equality $O_{\pi} K[G]=K[M]$, which yields, by restricting to $M \times 1$ in $M \times M$, another proof of the good filtration statement in the previous theorem. 
4.5. Corollary. Suppose the reductive normal algebraic monoid $M$ has 1-dimensional center $Z(M)$ and that $M$ has a zero element. As an $M \times M$-module under joint left and right translation, $K[M]$ has a filtration with sections of the form $H^{0}(\lambda) \otimes H^{0}\left(\lambda^{*}\right)$ for $\lambda \in X(\bar{T})^{+}$, each occurring exactly once.

Proof. This follows immediately from the equality $O_{\pi} K[G]=K[M]$, by Donkin [4, $(2.2 \mathrm{a})]$.

\section{Polynomial Representations}

Retain the assumptions and notations of the previous section. In particular, $M$ is a normal reductive affine algebraic monoid over $K$ with $\operatorname{dim} Z(M)=1$ and $0 \in M$ and $G$ is its unit group. We will identify $M$ with a closed submonoid of $\mathrm{M}_{n}$ in such a way that $\bar{T}$ consists of diagonal matrices. (This is always possible.) The assumption $\operatorname{dim} Z(G)=1$ means that, under the above identification, $G$ contains the subgroup

$$
\mathrm{H}_{n}=\left\{c I_{n}: c \in K^{\times}\right\}
$$

of scalar matrices. This means that $G$ admits a graded polynomial representation theory [8, Prop. 1.5] and all the results of [8] are applicable to $G$. In particular, the rational representation theory of $M$ is equivalent to the polynomial representation theory of $G[8$, Prop. 2.2]

We want to summarize the results of [8], but first we need to introduce more notation. Let $\mathrm{A}(n)$ denote the algebra of regular functions on $\mathrm{M}_{n}$; i.e., polynomials in the $n^{2}$ matrix entries $\mathrm{T}_{i j}, 1 \leq i, j \leq n$. Let $\mathrm{A}(n, d)$ denote the span of the $d$-fold products of the linear maps on $\mathbf{M}_{n}$. Elements of $\mathbf{A}(n, d)$ are homogeneous polynomials of degree $d$ in the generators $\left\{\mathrm{T}_{i j}\right\}$ and $\mathrm{A}(n)=\bigoplus_{d \geq 0} \mathrm{~A}(n, d)$. This is of course just the $\mathrm{H}_{n}$-eigenspace decomposition of the coordinate algebra $K\left[\mathrm{M}_{n}\right]$ under left or right translation. The coalgebra structure on $\mathrm{A}(n)$ is determined by

$$
\Delta\left(\mathrm{T}_{i j}\right)=\sum_{k=1}^{n} \mathrm{~T}_{i k} \otimes \mathrm{T}_{k j}, \quad \varepsilon\left(\mathrm{T}_{i j}\right)= \begin{cases}1 & \text { if } i=j, \\ 0 & \text { otherwise. }\end{cases}
$$

and $\mathrm{A}(n, d)$ is a subcoalgebra of $\mathrm{A}(n)$ for each $d$. Let $A(G), A_{d}(G)$ denote respectively the image of $\mathrm{A}(n), \mathrm{A}(n, d)$ under the restriction map $K\left[\mathrm{M}_{n}\right]$ to $K[G]$. The bialgebra $A(G)$ is the direct sum of the subcoalgebras $A_{d}(G)$ (that is the meaning of graded polynomial representation theory). By a polynomial $G$-module we mean a $G$-module with coefficients contained in $A(G)$; by a homogeneous polynomial $G$-module of degree $d$ we mean one with coefficients contained in $A_{d}(G)$.

Set $\mathrm{S}(n, d)=\mathrm{A}(n, d)^{*}=\operatorname{Hom}_{K}(\mathrm{~A}(n, d), K)$. Since $\mathrm{A}(n, d)$ is a coalgebra, $\mathrm{S}(n, d)$ is an (associative) algebra. The $\mathrm{S}(n, d)$ are known as Schur algebras. Set $S_{d}(G)=$ $A_{d}(G)^{*}$. Since $A_{d}(G)$ is a quotient of $\mathrm{A}(n, d)$, we may identify $S_{d}(G)$ with a subalgebra of the Schur algebra $\mathrm{S}(n, d)$. If we set $V=K^{n}$ with $G$ acting by left matrix multiplication, then $G$ acts diagonally on the tensor power $V^{\otimes d}$ and $S_{d}(G)$ is isomorphic with the enveloping algebra of $G$ (or of $M$ ) acting on $V^{\otimes d}$ [8, Prop. 3.2].

We have $K[M]=\bigoplus_{d \geq 0} K[M]_{d}$, the $\mathrm{H}_{n}$ (or even $\overline{\mathrm{H}}_{n}$ )-eigenspace decomposition for left or right translation. The restriction map $K[M] \rightarrow K[G]$ is injective and maps $K[M]$ onto $A(G), K[M]_{d}$ onto $A_{d}(G)$. Thus $K[M]_{d} \simeq A_{d}(G)$ as coalgebras, $K[M] \simeq A(G)$ as bialgebras. We have

5.1. Proposition. [8, Prop. 1.3, 1.4] 
(a) Every polynomial $G$-module $V$ has a direct sum decomposition of the form $V=$ $\bigoplus_{d \geq 0} V_{d}$ where each $V_{d}$ is homogeneous of degree $d$.

(b) The category of homogeneous polynomial $G$-modules of degree d is equivalent to the category of $S_{d}(G)$-modules.

In [4], Donkin introduces the notion of a generalized Schur algebra $S_{\pi}(G)$ associated to a reductive group $G$ and a finite ideal $\pi$ in $X(T)^{+}$. He defines $A_{\pi}(G)$ to be $O_{\pi} K[G]$ and shows this is a subcoalgebra of $K[G]$. Then $S_{\pi}(G)$ is defined to be its linear dual $A_{\pi}(G)^{*}$. We will now show that our algebras $S_{d}(G)$ are in fact generalized Schur algebras in Donkin's sense.

Restriction from $T$ to $\mathrm{H}_{n}$ gives a map $X(T) \rightarrow X\left(\mathrm{H}_{n}\right)$. Let us define a character $\chi_{d}$ on $\mathrm{H}_{n}$ by the rule $c I_{n} \mapsto c^{d}$ for any $d \in \mathbb{Z}, c \in K^{\times}$. Then $X\left(\mathrm{H}_{n}\right)=\left\{\chi_{d}: d \in \mathbb{Z}\right\}$ and we have

$$
X(T)=\bigcup_{d \in \mathbb{Z}} X(T)_{d} \quad \text { (disjointly) }
$$

where $X(T)_{d}$ is the inverse image of $\chi_{d}$ under the restriction map $X(T) \rightarrow X\left(\mathrm{H}_{n}\right)$. Moreover,

$$
\text { if } \lambda \in X(T)_{d}, \mu \in X(T)_{e} \text {, then } \lambda+\mu \in X(T)_{d+e} .
$$

Similarly, restriction from $\bar{T}$ to $\mathrm{H}_{n}$ gives a map $X(\bar{T}) \rightarrow X\left(\mathrm{H}_{n}\right)$. Defining $X(\bar{T})_{d}$ to be the inverse image of $\chi_{d}$ under this map, we have

$$
X(\bar{T})=\bigcup_{d \geq 0} X(\bar{T})_{d} \quad \text { (disjointly). }
$$

Moreover, our identification of $X(\bar{T})$ with a subset of $X(T)$ identifies $X(\bar{T})_{d}$ with $X(T)_{d}$, for all $d \geq 0$.

We will soon need the following fact.

5.2. Lemma. We have the inclusion $\Phi \subset X(T)_{0}$.

Proof. If $V$ is any rational $T$-module we have the $T$-weight space decomposition $V=\bigoplus_{\lambda \in X(T)} V_{\lambda}$ and the $T$-weights of $V$ are $\left\{\lambda \in X(T): V_{\lambda} \neq(0)\right\}$. Restricting to $\mathrm{H}_{n}$ we have $V=\bigoplus_{d \in \mathbb{Z}} V_{d}$, where $V_{d}$ is the $\chi_{d}$-eigenspace. Moreover, restriction to $\mathrm{H}_{n}$ maps the $T$-weights of $V$ onto the $\mathrm{H}_{n}$-weights of $V$. If $T$ acts by conjugation, then the restricted $\mathrm{H}_{n}$-action is trivial, so in that case $V=V_{0}$ and the $T$-weights of $V$ will all lie in $X(T)_{0}$. Specializing $V$ to be the Lie algebra $\mathfrak{g}$ of $G$, with $G$ acting via the adjoint representation, thus yields the result.

5.3. Proposition. For each $d \geq 0, X(\bar{T})_{d}^{+}$is a finite ideal in the poset $X(T)^{+}$.

Proof. Suppose $\mu \leq \lambda$ for $\lambda \in X(\bar{T})_{d}^{+}, \mu \in X(T)^{+}$. Then $\mu \in X(\bar{T})^{+}$, by 3.6. By (14) and 5.2 we have $\lambda-\mu \in X(T)_{0}$; hence $\mu \in X(T)_{d}^{+}$and consequently we have $\mu \in X(\bar{T})_{d}^{+}$. This shows $X(\bar{T})_{d}^{+}$is an ideal in the poset $X(T)^{+}$.

To see that it is finite, recall that we may assume that $T$ consists of diagonal matrices. So we have a commutative diagram

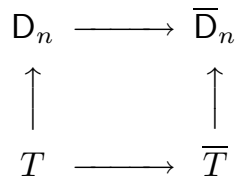


in which all the maps are inclusions. This in turn induces the commutative diagram

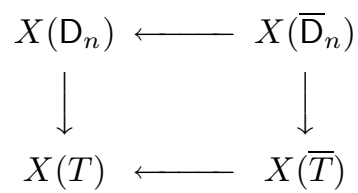

in which all the maps are restriction maps. The horizontal maps are injective (see Section 3) and the vertical maps are surjective. (See [1, Prop. 8.2(c)] for the surjectivity of the vertical map on the left; for the surjectivity of the vertical map on the right see the proof of [12, Lemma 2.2].)

Now it is very easy to check that $X\left(\overline{\mathrm{D}}_{n}\right)_{d}$ is finite for each $d \geq 0$. Since restriction carries $X\left(\overline{\mathrm{D}}_{n}\right)_{d}$ onto $X(\bar{T})_{d}$ it follows that $X(\bar{T})_{d}$ is finite. Hence $X(\bar{T})_{d}^{+}$is finite.

We now obtain a generalization of $[5,(1)$, p. 356]:

5.4. Theorem. Suppose the reductive normal algebraic monoid $M$ has 1-dimensional center $Z(M)$ and that $M$ has a zero element. With $\pi(d)=X(\bar{T})_{d}^{+}$we have the equalities $A_{d}(G)=O_{\pi(d)} K[G], S_{d}(G)=S_{\pi(d)}(G)$. In particular, $S_{d}(G)$ is a generalized Schur algebra.

Proof. We have an isomorphism $A_{d}(G) \simeq K[M]_{d}$ and

$$
K[M]_{d}=\bigoplus_{\lambda \in X(\bar{T})_{d}} K[M]_{\lambda}
$$

By an argument similar to the one in the proof of 4.4 we see that $O_{\pi(d)}$ maps $\operatorname{ind}_{T}^{G} K_{\lambda}$ onto $\operatorname{ind} \frac{M}{T} K_{\lambda}$ for $\lambda \in X(\bar{T})_{d}$ and to (0) otherwise. Thus it follows that $O_{\pi(d)} K[G]=A_{d}(G)$. The equality $S_{d}(G)=S_{\pi(d)}(G)$ follows immediately.

5.5. Corollary. Suppose the reductive normal algebraic monoid $M$ has 1-dimensional center $Z(M)$ and that $M$ has a zero element.

(a) The category of $S_{d}(G)$-modules is a highest weight category, in the sense of Cline, Parshall, Scott [2].

(b) For each $d \geq 0, S_{d}(G)$ is a quasihereditary algebra with global dimension bounded by $2 \ell\left(X(\bar{T})_{d}^{+}\right)$.

(c) The $K$-vector space dimension of $S_{d}(G)$ is given by the formula

$$
\operatorname{dim} S_{d}(G)=\sum_{\lambda \in \pi(d)}\left(\operatorname{dim} H^{0}(\lambda)\right)^{2} .
$$

(d) If $V, W$ are homogeneous polynomial $G$-modules of degree $d$, then

$$
\operatorname{Ext}_{M}^{\bullet}(V, W) \simeq \operatorname{Ext}_{G}^{\bullet}(V, W) \simeq \operatorname{Ext}_{S_{d}(G)}^{\bullet}(V, W) .
$$

Proof. (a) follows from what has already been proven. The first statement of (b) follows from (a) by the main result of [2], and the second statement from (2.2f) in [4]. For (c) and (d) see (2.2c) and (2.2d) in [4].

\section{REFERENCES}

[1] A. Borel, Linear Algebraic Groups, 2nd ed., Springer-Verlag, 1991. MR 92d:20007

[2] E. Cline, B. Parshall, and L. Scott, Finite dimensional algebras and highest weight categories, J. Reine Angew. Math. 391 (1988), 85-99. MR 90d:18005

[3] M. Demazure and P. Gabriel, Introduction to Algebraic Geometry and Algebraic Groups, North-Holland, 1980. MR 82e:14001 
[4] S. Donkin, On Schur algebras and related algebras I, J. Algebra 104 (1986), 310-328. MR 89b:20084a

[5] S. Donkin, On Schur algebras and related algebras II, J. Algebra 111 (1987), 354-364. MR 89b:20084b

[6] S. Donkin, Good filtrations of rational modules for reductive groups, Proc. Symposia Pure Math. (Part I) 47 (1987), 69-80. MR 89f:20048

[7] S. R. Doty, Resolutions of B-modules, Indag. Math. N.S. 5 (1994), 267-283. MR 95h:20053

[8] S. R. Doty, Polynomial representations, algebraic monoids, and Schur algebras of classical type, J. Pure Applied Algebra 123 (1998), 165-199. MR 98j:20057

[9] E. Friedlander and A. Suslin, Cohomology of finite group schemes over a field, Invent. Math. 127 (1997), 209-270. MR 98h:14055a

[10] J. E. Humphreys, Lie Algebras and Representation Theory, (second printing, revised) Springer-Verlag, 1972. MR 48:2197

[11] J. E. Humphreys, Linear Algebraic Groups, Springer-Verlag, 1975. MR 53:633

[12] M. Putcha, On linear algebraic semigroups III, Internat. J. Math. EJ Math. Sci. 4 (1981), 667-690. MR 83k:20073a

[13] M. Putcha, Linear Algebraic Monoids, London Math. Soc. Lecture Notes Series 133, Cambridge Univ. Press, 1988. MR 90a:20003

[14] L. Renner, Classification of semisimple algebraic monoids, Trans. Amer. Math. Soc. 292 (1985), 193-223. MR 86b:20052

[15] L. Renner, Conjugacy classes of semisimple elements and irreducible representations of algebraic monoids, Commun. in Algebra 16 (1988), 1933-1943. MR 89e:20115

[16] L. Solomon, An introduction to reductive monoids, in Semigroups, formal languages and groups (York, 1993), 295-352, NATO Adv. Sci. Inst. Ser. C Math. Phys. Sci., 466, Kluwer Acad. Publ., Dordrecht, 1995. CMP 98:15

Mathematical and Computer Sciences, Loyola University of Chicago, Chicago, IlliNOIS 60626

E-mail address: doty@math.luc.edu 